\title{
ACCELERATION OF CONVERGENCE OF REGULAR PERTURBATION SERIES
}

\author{
M. A. SOLIMAN \\ Department of Chemical Engineering, University of Riyad, P. O. Box 800, Riyad, Saudi Arabia
}

\section{Introduction}

Regular perturbation techniques have been used for obtaining approximate solutions of nonlinear problems, in the form of a power series in terms of a small parameter. Only a few terms can be obtained analytically. Using the computer, we can obtain a large number of terms. This is called computer extension of the series. This is not always an easy task. Suzuki ${ }^{4}$ has recently suggested the use of invariant theory for obtaining higher-order terms for problems described by partial differential equations. Most research workers in chemical engineering used to terminate the analysis by obtaining a series valid for some range, usually small, for the parameter of interest. We would like in this communication to draw attention to a group of techniques that can be used in improving the convergence of the series obtained ${ }^{5}$. We introduce in this paper two of these techniques, Pade approximants and the extraction of additive singularity. We will try these techniques on the series obtained by Suzuki ${ }^{4)}$ for two diffusion problems. For other techniques and applications in fluid mechanics, heat transfer, and chemical reaction engineering, we refer the reader to references ${ }^{1,3,5)}$.

\section{Illustration of Techniques}

\section{1 Diffusion with chemical reaction}

Suzuki ${ }^{4}$ obtained the following series for the enhancement factor $E$ for diffusion with first-order chemical reaction in semi-infinite medium.

$E=\varepsilon\left(1+\varepsilon-\frac{1}{6} \varepsilon^{2}+\frac{1}{30} \varepsilon^{3}-\frac{1}{168}-\varepsilon^{4}+\frac{1}{1080} \varepsilon^{5}-\ldots\right)$

where $\varepsilon$ is a small parameter representing dimensionless time. We notice that except for the first two terms, the sign of the series alternates. This means that the singularity of the series lies on the negative axis and thus has no physical meaning. With few terms given, we prefer to use Pade approximants. Here we recast the series in the form of a ratio of two polynomials $M$, $N$ of equal or different orders, written as $[M / N]$ Pade

Received October 6, 1980 . approximant. The behaviour of $E$ as $\varepsilon \rightarrow \infty$ can be incorporated in the selection of the degrees of the polynomials. In our case it can be shown from the analytical solution of Danckwerts ${ }^{2}$ that as $\varepsilon \rightarrow \infty E$ tends to $\sqrt{\pi \varepsilon}$ or $(E / \varepsilon)^{2} \rightarrow \pi / \varepsilon$. Thus it is better to obtain first the series for $(E / \varepsilon)^{2}$ and then choose a Pade approximent such that the order of the numerator polynomial is one degree less than that of the denominator. Thus, we have

$$
(E / \varepsilon)^{2}=1-\varepsilon / 3+\frac{17}{180} \varepsilon^{2}-\frac{29}{260} \varepsilon^{2}+\frac{187}{37800} \varepsilon^{4}-\ldots
$$

Using a [1/2] Pade approximant, we obtain

$$
(E / \varepsilon)^{2}=(1+11 / 63 \varepsilon) /\left(1+\frac{32}{63} \varepsilon+\frac{283}{3780} \varepsilon^{2}\right)
$$

Here $(E / \varepsilon)^{2} \rightarrow 660 / 283 \varepsilon$ which is close to $\pi / \varepsilon$. From Danckwerts' analytical solution ${ }^{2 y}$ we obtain the following expression for the enhancement factor $E$ :

$$
E=\exp (-\varepsilon)-1+\sqrt{\pi \varepsilon} \operatorname{erf}(\sqrt{\varepsilon})
$$

In Tbble 1, we compare solutions obtained from straight-forward expansion (1), Pade approximant from equation (3) and the analytical solution. It is noticed that the straight-forward expansion (1) is useless for $\varepsilon>4$ whereas the series in the form of Pade approximant (3) is in reasonable agreement (less than $10 \%$ error) with the analytical solution for all $\varepsilon$. We could make this agreement better by considering more terms.

\subsection{Diffusion with variable diffusivity}

Suzuki $^{4}$ obtained the following expression for the instantaneous flux $F$ for a transient diffusion problem with exponential diffusivity,

$$
\begin{aligned}
F & =\sum_{n=0}^{\infty} c_{n} \varepsilon^{n}=-(1.12837917+0.20501534 \varepsilon \\
& +0.08897813 \varepsilon^{2}+0.05054114 \varepsilon^{3}+0.03287074 \varepsilon^{4} \\
& +0.02321012 \varepsilon^{5}+0.01732255 \varepsilon^{8}+0.01345554 \varepsilon^{7} \\
& +0.01077242 \varepsilon^{8}+0.00883088 \varepsilon^{9}+0.00737851 \varepsilon^{10} \\
& 0.00626239 \varepsilon^{11}+0.00538530 \varepsilon^{12}+0.00468300 \varepsilon^{13} \\
& \left.0.00411156 \varepsilon^{14}+0.00364009 \varepsilon^{15}+\ldots\right)
\end{aligned}
$$

where $\varepsilon$ is a small parameter representing the variability of the diffusivity. Here, we have enough terms 
Table 1 Comparison of solutions for the diffusion with chemical reaction problem

\begin{tabular}{rccc}
$\varepsilon$ & Eq. (1) & Eq. (3) & Exact solution(4) \\
\hline 1 & 0.86164 & 0.86145 & 0.86153 \\
2 & 1.53439 & 1.52673 & 1.52791 \\
4 & 2.89101 & 2.53472 & 2.54664 \\
5 & 4.17328 & 2.94162 & 2.96386 \\
10 & 59.7355 & 4.49909 & 4.60499 \\
20 & 2230.58 & 6.61152 & 6.92666 \\
50 & 255949. & 10.6724 & 11.5331 \\
100 & 8695790. & 15.1861 & 16.7245 \\
\hline
\end{tabular}

to try Van Dyke's ${ }^{5}$ scheme consisting of three steps. The first step is computer extension of the series which has been carried out by Suzuki in the form of Eq. (5). The second step is to locate the singularity using a Domb-Sykes plot, which is a plot of the ratio of the subsequent terms $c_{n} / c_{n-1}$ against $1 /(n+\delta)$ where $\delta$ is a number which makes the plot of $c_{n} / c_{n-1}$ for large $n$ against $1 /(n+\delta)$ linear. As shown in Fig. 1, the plot is somewhat curved for $\delta=0$. For $\delta=1$, the curve becomes linear for large $n$ and unmistakably tends to a value of $\left(c_{n} / c_{n-1}\right)_{n \rightarrow \infty}=1$. This is in fact the reciprocal of the radius of the convergence $\varepsilon_{0}$ of the primary singularity of the series. The singularity here lies on the positive axis and thus has some physical meaning; which is for $\varepsilon>1$, we have negative diffusivity. Our series is thus very slowly convergent for all $0 \leq \varepsilon \leq 1$. We can now improve its convergence by carrying out the third step, which is the extraction of singularity. We can extract the primary singularity in the form

$$
\begin{aligned}
f(\varepsilon) & =\sum_{n=0}^{\infty} d_{n} \varepsilon^{n} \\
& =\text { constant } \times \begin{cases}\left(\varepsilon_{0} \pm \varepsilon\right)^{\alpha} & \alpha \neq 0,1,2 \\
\left(\varepsilon_{0} \pm \varepsilon\right)^{\alpha} & \log \left(\varepsilon_{0}+\varepsilon\right) \quad \alpha=0,1,2\end{cases}
\end{aligned}
$$

since for large $n$ the Domb-Sykes plot becomes linear, that is

$$
\frac{d_{n}}{d_{n-1}}=\mp-1-\frac{1+\alpha}{\varepsilon_{0}}\left(1-\frac{1}{n}\right)
$$

Thus the exponent $\alpha$ is determined from the relation

$$
\alpha=-(1+\text { slope of the plot })
$$

For our case $\alpha=0.8$. Instead of using the form (6) as suggested by Van Dyke ${ }^{5}$, we extract the singularity in the form

$$
f(\varepsilon)=\mathrm{constant} \times\left[\left\{\left(\varepsilon_{0}+\varepsilon\right)^{\alpha}-\varepsilon_{0}^{\alpha}\right\} / \alpha \varepsilon\right]
$$

since now we have

$$
-\frac{d_{n}}{d_{n-1}}=\mp \frac{1}{\varepsilon_{0}}\left(1-\frac{1+\alpha}{n+1}\right)
$$

Thus for this new form the plot is actually linear against $1 /(n+1)$.

We can now factor out the following function from the series (5),

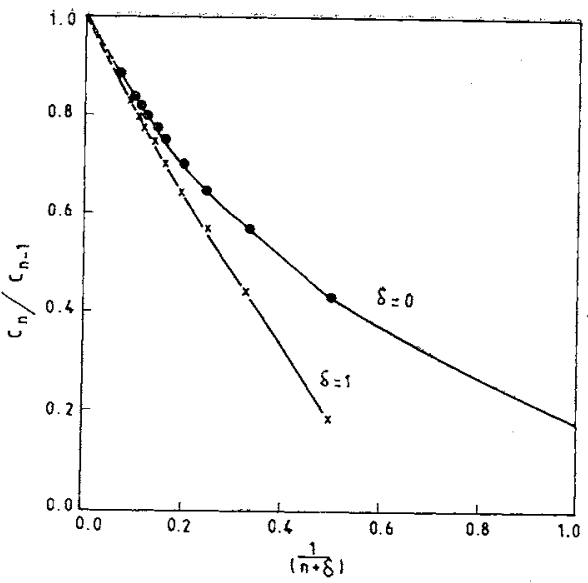

Fig. 1 Domb-Sykes plot for example 2

$$
f(\varepsilon)=A\left[(1-\varepsilon)^{0.8}-1\right] /(0.8 \varepsilon)
$$

we can do that either by dividing the series over $f(\varepsilon)$ or subtracting it from $f(\varepsilon)$. With positive exponent, it is better to extract the singularity by subtraction. In this case, we have to determine the amplitude of the singularity $A$. We determine A such that coefficient of the last term in the series becomes null. Thus

$A=0.00364009 \times(16) ! \Gamma(0.2) / \Gamma(15.2)=2.356004$

where $\Gamma$ is the gamma function.

The final form of the series becomes

$$
\begin{aligned}
F= & 2.945005\left[(1-\varepsilon)^{0.8}-1\right] / \varepsilon \\
& +1.2276248+0.02958511 \varepsilon+0.00486206 \varepsilon^{2} \\
& +0.00107097 \varepsilon^{3}+0.00016101 \varepsilon^{4}-0.00008789 \varepsilon^{5} \\
& -0.00014604 \varepsilon^{6}-0.00014374 \varepsilon^{7}-0.00012299 \varepsilon^{8} \\
& -0.00009834 \varepsilon^{9}-0.00007493 \varepsilon^{10}-0.00005435 \varepsilon^{11} \\
& -0.00003683 \varepsilon^{12}-0.00002219 \varepsilon^{13}-0.00001005 \varepsilon^{14}
\end{aligned}
$$

It is clear that the coefficients have been greatly reduced. It is very probable that the new series (13) is accurate for the whole physical range of $0 \leq \varepsilon \leq 1$.

Nomenclature

$$
\begin{array}{ll}
A & =\text { amplitude } \\
E & =\text { enhancement factor } \\
F & =\text { instantaneous flux } \\
\alpha & =\text { exponent } \\
\varepsilon_{0} & =\text { radius of convergence of a series } \\
\varepsilon & =\text { small parameter }
\end{array}
$$

\section{Literature Cited}

1) Aziz, A.: Trans. ASME, J. of Heat Transfer, in press.

2) Danckwerts, P. V.: Trans. Farad. Soc., 46, 300 (1950).

3) Soliman, M. A.: Submitted to Computers and Chemical Engineerning.

4) Suzuki, M.: J. Chem. Eng. Japan, 12, 400 (1979).

5) Van Dyke, M.: Q.J. Mech. Appl. Math., 27, 423 (1974). 